\title{
Globular Clusters and Planetary Nebula Kinematics in NGC 5128 (Cen A)
}

\author{
Eric W. Peng
}

The Johns Hopkins University, Department of Physics and Astronomy, Baltimore, MD, 21218, USA

Holland C. Ford

The Johns Hopkins University, Department of Physics and Astronomy, Baltimore, $M D$, 21218, USA

Kenneth C. Freeman

RSAA, Australian National University, Canberra, Australia

\begin{abstract}
We have conducted a wide-field optical survey of NGC 5128 (Cen A), the nearest large elliptical galaxy $(3.5 \mathrm{Mpc})$. From $U B V R I$ imaging and spectroscopic follow-up, we have identified and obtained kinematics for 125 new globular clusters out to a projected radius of $40 \mathrm{kpc}$. We also extend our survey of field star kinematics in NGC 5128, and now possess a total of 736 radial velocities for planetary nebulae out to a projected radius of $80 \mathrm{kpc}$.
\end{abstract}

- The PNe sample along the photometric major axis shows significant rotational support out to $80 \mathrm{kpc}$.

- The blue globular clusters seem to possess little or no angular momentum.

- The kinematics of the red globular cluster sample show some evidence for rotation around an axis common to that of the field stars.

\section{Introduction}

The inter-relationship between star clusters and field stars has provided interesting constraints on the formation of galaxies. Comparisons of the kinematics of these two stellar populations is relatively unexplored, especially in the outer halos of galaxies, and can provide insight on their respective origins. In an effort to address these issues, we are conducting an imaging and spectroscopic survey of globular clusters (GCs) and planetary nebulae (PNe) in NGC 5128 (Cen A), the nearest large elliptical galaxy. 


\section{Observations, Selection, and Data}

Our data consists of three components: 1) UBVRI imaging of NGC 5128 taken with the CTIO Mosaic camera. The fields cover halo regions out to $50 \mathrm{kpc}$ along the photometric major axis, and $30 \mathrm{kpc}$ along the photometric minor axis. The selection and depth of these fields allows a study of the galaxy's globular cluster population, as well as its faint unresolved field star light. These fields are shown in Figure 1a. 2) [OIII] imaging out to $100 \mathrm{kpc}$ and $50 \mathrm{kpc}$ along the photometric major and minor axes, respectively. These fields are outlined in Figure 1b. 3) Fiber spectroscopy of GC and PN candidates. These were obtained in January and February, 2001, with both the 2dF at AAO, and Hydra at CTIO. The faintest clusters we observe have $V \sim 21$, with the mean of the distribution being around $V \sim 19.5$.

While NGC 5128's proximity makes its cluster population relatively accessible, its low galactic latitude $\left(+19^{\circ}\right)$ makes foreground star contamination a serious problem. Clusters are slightly resolved in our images, and candidates were selected on sharpness and size. Color was also used to eliminate some background galaxies and foreground stars. Our GC yield, as determined from spectroscopic follow-up is $\sim 52 \%$. We identified 125 new GCs out to a projected radius of $\sim 40 \mathrm{kpc}$. Added to the sample of Harris et al. (1992), these give a total of 184 confirmed GCs in NGC 5128.

Planetary nebulae are readily identified using narrowband imaging of the [OIII] $\lambda 5007$ line, combined with imaging in an off-band (continuum). We measure radial velocities with spectroscopy of the same bright [OIII] emission line. Since the study of Hui et al. (1995) in which velocities were obtained for 433 PNe in NGC 5128, we have added 303 more PNe, most of which are in the halo regions between radii of 20 and $80 \mathrm{kpc}$.

\section{Kinematics}

Preliminary results from photometry and radial velocities of GCs and PNe show that: 1) The previously detected bimodality in the $V-I$ color distribution of GCs is confirmed. 2) The field star population as traced by the PNe contains significant amounts of angular momentum, especially in the halo. 3) The blue clusters, $(V-I)_{0}<0.98$, show little rotation. 4) The red clusters, $(V-I)_{0}>0.98$, show weaker, but possible rotation about the same kinematic axis as the PNe.

\section{References}

Harris, G., Geisler, D., Harris, H., \& Hesser, J. 1992, AJ, 287, 175

Hui, X., Ford, H. C., Freeman, K. C. \& Dopita, M. A. 1995, ApJ, 449, 592 

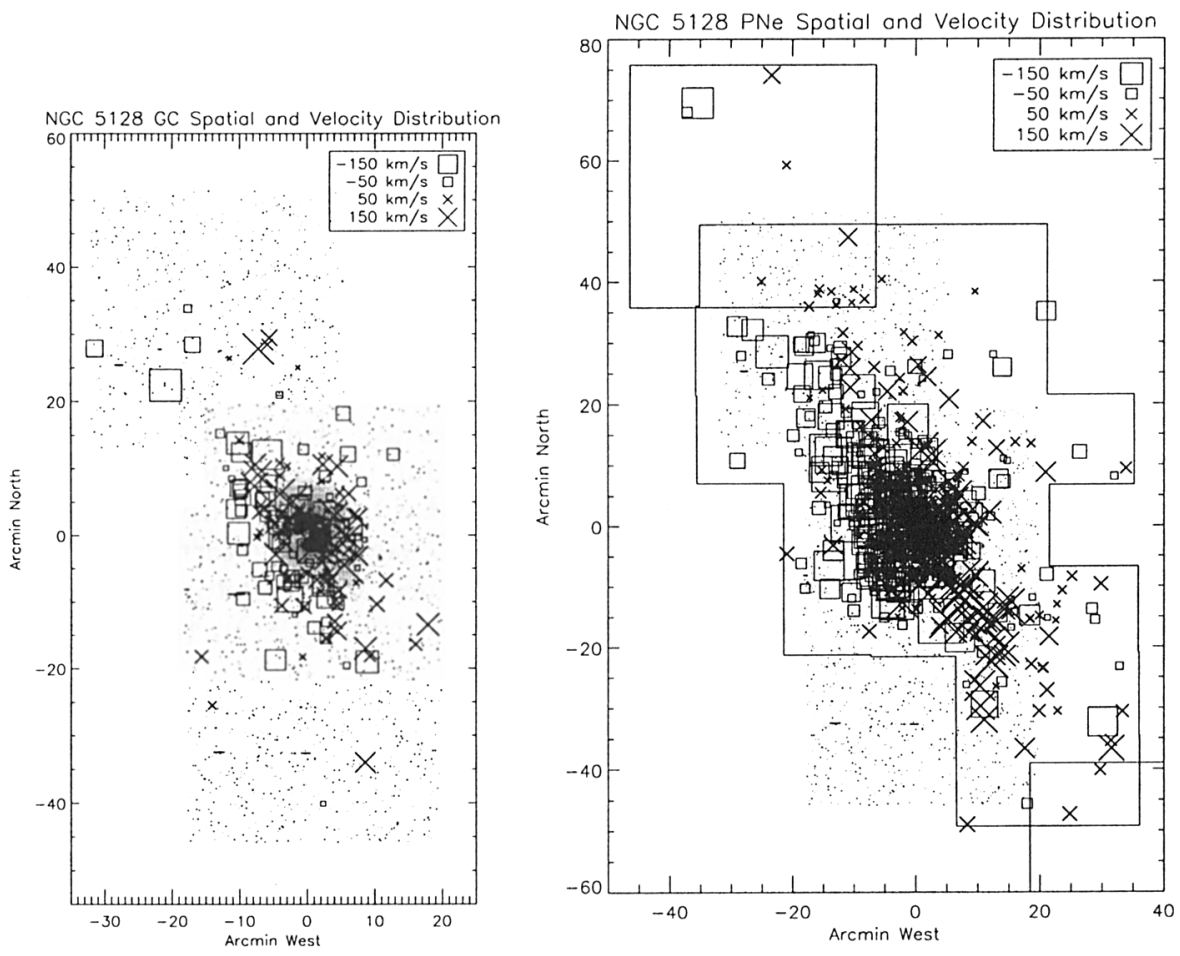

Figure 1. Figure 1a (left). Globular Clusters in NGC 5128. 184 confirmed globular clusters (59 from H92, and 125 from our survey) are plotted on our three Mosaic pointings. The velocities in the legend are with respect to the systemic velocity of NGC 5128, which we take to be $541 \mathrm{~km} / \mathrm{s}$. The symbols represent the direction of the radial velocities with respect to the galaxy, with crosses going into the page, and squares coming out. The size of the point is proportional to the absolute deviation from systemic velocity. The physical scale at the distance of NGC 5128 is $1^{\prime}=1.02 \mathrm{kpc}$. North is up, and east is to the left. Figure 1b (right). 736 Planetary Nebulae in NGC 5128. Confirmed $\mathrm{PNe}$ and their velocities are overplotted on our Mosaic images. The actual area surveyed for $\mathrm{PNe}$, outlined in black, substantially overlaps and goes beyond our cluster fields. Our most distant PNe in the north-east are at $\sim 80 \mathrm{kpc}$ in projected radius. The symbols and sizes for points follows the scheme in Figure 1a. Rotation along the major axis is evident, with the NE side approaching and the SW side receding. 